\title{
Anaphylaxis as a Side Effect of Pantoprazole
}

\author{
Hesam Yousefi ${ }^{1}$, Siamak Moayedi ${ }^{2}$, Mehdi Harorani $^{3}$, Ali Sahebi ${ }^{4}$ and Mohamad Golitaleb ${ }^{3,{ }^{*}}$ \\ ${ }^{1}$ Department of Pharmaceutical Management, Sajad Hospital, Kermanshah, Iran \\ ${ }^{2}$ Department of Emergency Medicine, University of Maryland School of Medicine, Baltimore, USA \\ ${ }^{3}$ Department of Nursing, School of Nursing and Midwifery, Arak University of Medical Sciences, Arak, Iran \\ ${ }^{4}$ Clinical Research Development Unit, Shahid Mostafa Khomeini Hospital, Ilam University of Medical Sciences, Ilam, Iran \\ "Corresponding author: Department of Nursing, School of Nursing and Midwifery, Arak University of Medical Sciences, Arak, Iran. Email: mohamadgolitaleb@gmail.com
}

Received 2019 August 25; Revised 2019 September 07; Accepted 2019 September 11.

Keywords: Allergic Reaction, Pantoprazole, Anaphylaxis, Proton Pump Inhibitors, Hypersensitivity

\section{Dear Editor,}

Proton pump inhibitors (PPIs) block gastric acid secretion by inhibiting the hydrogen/potassium adenosine triphosphatase enzyme system. These drugs are used to treat gastric ulcers, esophagitis, duodenal ulcers, Zollinger-Ellison syndrome, Barrett's esophagus, and gastroesophageal reflux disease $(1,2)$. Although PPIs have acceptable safety, their administration can result in allergic and anaphylactic reactions. Although these reactions, either immediate or delayed, are unusual, they can be life threatening. A number of publications have reported anaphylactic reactions after administration of PPIs. The actual rate of adverse reactions to these drugs is unknown (3-5). We describe a case of anaphylactic reaction to an intravenous administration of pantoprazole in a patient treated for a presumed peptic ulcer. A 45-year-old male was presented to our Emergency Department complaining of four days of increasing epigastric pain. The pain was limited to the epigastric area without radiation. The pain was improved immediately after eating meals, however, it intensified 30 minutes afterwards. The patient had defecated twice daily without melena and hematochezia. He did not have any vomiting. He was mildly tender to the palpation of the epigastric region. The patient and her family had no history of allergic diseases and drug hypersensitivity. In addition, the patient declared that he had consumed nothing before taking Pantazole. The patient's vital signs at the time of admission were as follows: heart rate $(\mathrm{HR})=$ $88 \mathrm{bpm}$, respiratory rate $(\mathrm{RR})=17 \mathrm{bpm}$, oxygen saturation $\left(\mathrm{O}_{2} \mathrm{Sat}\right)=95 \%$, and blood pressure $(\mathrm{BP})=140 / 95 \mathrm{mmHg}$. The patient was diagnosed with a presumed peptic ulcer. He was treated with a $40 \mathrm{mg}$ intravenous does of pantoprazole. After three minutes of injection initiation, the patient developed hives, itching, erythema of the upper extremi- ties, angioedema, hypotension, cyanosis, and dyspnea. The vital signs were as follows: $\mathrm{HR}=116 \mathrm{bpm}, \mathrm{RR}=8 \mathrm{bpm}, \mathrm{O}_{2}$ Sat $=75 \%$ and $\mathrm{BP}=80 / 60 \mathrm{mmHg}$.

The anaphylactic shock was managed by infusing normal saline (2 liters), intramuscular epinephrine (0.5 mg), intravenous hydrocortisone (200 mg), intravenous chlorpheniramine (a first-generation anit-histamine) (4 mg), and finally oxygen (8 liters per minute through a simple mask). The patient's general condition gradually recovered and completely improved after one hour. He was discharged after 24 hours. Anaphylaxis is a life-threatening systemic and allergic reaction affecting multiple organs including the cardiovascular and respiratory systems. This reaction is triggered by degranulation of mast cells and basophils. Foods, mediations, and insect venom are the most common causes of anaphylactic reactions. Delay in the treatment of anaphylaxis can lead to hypoxia, ischemia, encephalopathy, and even death $(6,7)$. In general PPIs are well tolerated. Common reactions of PPIs include headache, diarrhea, nausea, and dizziness (8). Rare serious reactions include anaphylaxis, Stevens-Johnson syndrome, toxic epidermal necrolysis, and renal and hepatic impairment (1, $2,7)$. Acute and delayed allergic reactions, as well as systemic manifestations, have been described following both oral and intravenous administration of PPIs $(1,2,7,9)$. Faridaalaee and Ahmadian Heris (8), reported anaphylactic reaction in a 21-year-old woman with gastritis hospitalized in the emergency ward following intravenous injection of 40 mg pantazole. The patient was discharged 12 hours after the reaction. In another report from Turkey, Kepil Ozdemir et al. (9), describe 60 patients who had developed hypersensitivity reactions following the oral administration of PPIs. In this report, anaphylactic reactions were observed in $20 \%$ of the patients who received $40 \mathrm{mg}$ pantoprazole 
tablets. Allergic reactions are rare following the administration of PPIs such as pantoprazole. Nevertheless, as these reactions can be life threatening, it is essential for emergency medicine providers to be aware of this side effect of pantoprazole and carefully monitor patients during and after injection of this medication.

\section{Footnotes}

Conflict of Interests: The authors have no conflict of interests.

Funding/Support: This study was conducted without any funding.

Informed Consent: The patient provided written informed consent for the publication.

\section{References}

1. Casciaro M, Navarra M, Inferrera G, Liotta M, Gangemi S, Minciullo PL. PPI adverse drugs reactions: A retrospective study. Clin $\mathrm{Mol} \mathrm{Al}$ lergy. 2019;17:1. doi: 10.1186/s12948-019-0104-4. [PubMed: 30675130]. [PubMed Central: PMC6337765].

2. Ramirez E, Cabanas R, Laserna LS, Fiandor A, Tong H, Prior N, et al. Proton pump inhibitors are associated with hypersensitivity reactions to drugs in hospitalized patients: A nested case-control in a retrospective cohort study. Clin Exp Allergy. 2013;43(3):344-52. doi: 10.1111/cea.12034. [PubMed: 23414543].

3. Demir S, Erdenen F, Gelincik A, Unal D, Olgac M, Coskun R, et al. Evaluation of the potential risk factors for drug-induced anaphylaxis in adult patients. Int Arch Allergy Immunol. 2019;178(2):167-76. doi: 10.1159/000494130. [PubMed: 30448840].

4. Benito-Garcia F, Chambel M, Morais-Almeida M. Anaphylaxis due to proton pump inhibitors: Current understanding and important clinical considerations. Expert Rev Clin Immunol. 2018;14(8):653-6. doi: 10.1080/1744666X.2018.1505504. [PubMed: 30058396].

5. Rekabi M, Arshi S, Darougar S, Rekabi V, Nabavi M, Fallahpour M, et al. Oral wheat immunotherapy in a patient with anaphylaxis despite Negative Sensitization tests. Shiraz E-Med J. 2018;20(2). e83309. doi: 10.5812/semj.83309.

6. Otani IM, Banerji A. Immediate and delayed hypersensitivity reactions to proton pump inhibitors: Evaluation and management. Curr Allergy Asthma Rep. 2016;16(3):17. doi: 10.1007/s11882-016-0595-8. [PubMed: 26810177].

7. Alolabi R, Liem JJ. Pantoprazole-induced anaphylaxis. J Allergy Clin Immunol.2019;143(2):AB73. doi: 10.1016/j.jaci.2018.12.227.

8. Faridaalaee G, Ahmadian Heris J. Anaphylaxis as a rare side effect of pantoprazole; a case report. Emerg (Tehran). 2018;6(1). e34. [PubMed: 30009236]. [PubMed Central: PMC6036523].

9. Kepil Ozdemir S, Yilmaz I, Aydin O, Buyukozturk S, Gelincik A, Demirturk $M$, et al. Immediate-type hypersensitivity reactions to proton pump inhibitors: Usefulness of skin tests in the diagnosis and assessment of cross-reactivity. Allergy. 2013;68(8):1008-14. doi: 10.1111/all.12189. [PubMed: 23895584]. 